\title{
The Development of API Manufacturing Processes - Targets and Strategies
}

\author{
Dierk Wieckhusen*
}

\begin{abstract}
This article gives an overview on the questions to be asked and the activities to be planned during the development of an API manufacturing process. First of all the API properties required for formulation such as purity, polymorphism and solvate formation, residual solvents, particle morphology and particle size distribution have to be defined. Then process parameters have to be evaluated that ensure that the drug substance with these properties can be produced on lab scale. The transfer to large-scale production is discussed with emphasis on scale-up effects, appropriate equipment, potential bottlenecks, and cost aspects. Finally practical hints are given as how to design an API crystallization and work-up process in a way that all given requirements for the API are fulfilled and the process can be run reproducibly and cost-effective in production.
\end{abstract}

Keywords: API · Drug formulation · Powder properties · Process chemistry · Prozess rubustness · Scale-up

\section{Introduction}

Developing final process steps of an API synthesis very literally means building a bridge between chemical development where the API is synthesized and pharmaceutical development where the active drug substance is formulated into a drug product as indicated in the flowchart in Fig. 1.

The last chemical step is usually a final purification by crystallization, sometimes combined with the formation of a salt. In this step, the relevant powder properties of the drug substance are defined that are of fundamental importance for the pharmaceutical formulation process. API manufacturing combines the unit operations crystallization, filtration and drying and to a certain extent also milling.

Among the unit operations outlined above, crystallization is by far the most important operation because the drug substance particles are formed in this step. Crystallization not only needs to provide the correct polymorphic or solvated form robustly and in acceptable yield, purity and throughput, it must also provide proper powder properties that govern the dry formulation process, such as average particle size, particle size distribution, flowability, shape, bulk density, etc.

It is important to note that filtration, and especially drying in agitated dryers, may have a substantial effect on the powder properties of the API, e.g. through abrasion. Special attention needs to be paid to the scale-up of filtration and drying, as these process steps frequently become the bottleneck in the production line with production figures of several hundreds of tons per year. It is therefore vital to assess filtration and drying characteristics at a very early stage of development in order to recognize potential issues, and to take appropriate measures to resolve them.
Arguably, reproducibility is the most crucial feature of the whole API manufacturing process - nothing is worse for pharmaceutical production than changing and unpredictable API qualities. Hence the main goal in chemical development of an API is the design of a robust manufacturing process that yields the desired powder characteristics at all times. This paper is not intended to be a review of the available scientific literature on downstream processing of pharmaceutical substances, but aims at providing insights into the questions that have to be raised during the development of crystallization, filtration and drying processes for APIs based on the experience of more than 50 successful development projects the author has participated in.

${ }^{\star}$ Correspondence: D. Wieckhusen

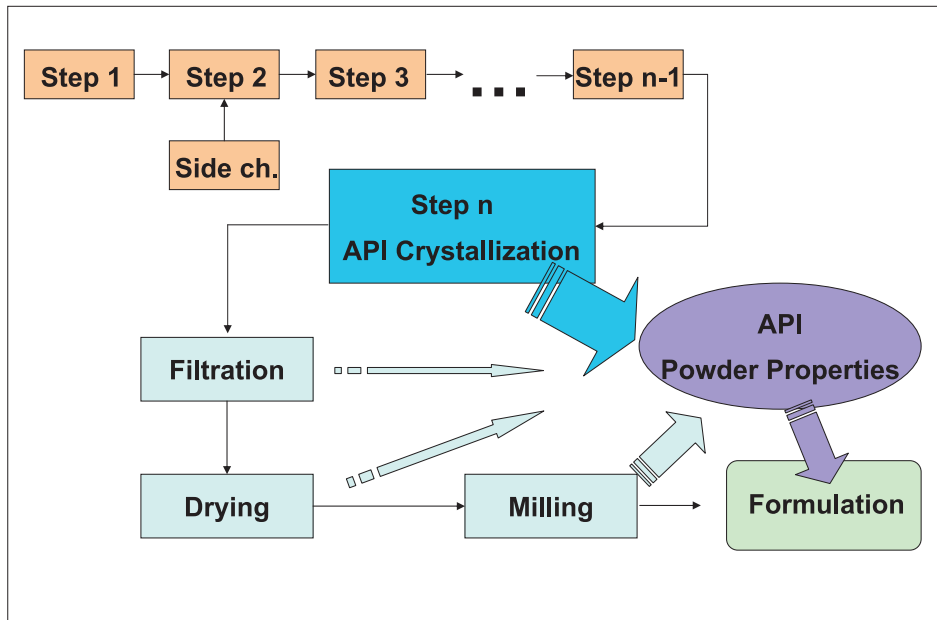

Fig. 1. API manufacturing flowchart 


\section{Defining the Goals for Process Development}

Because the process chemist does not design an API manufacturing process for himself but for his customers, it is worthwhile thinking about their expectations. The major customer is the pharmacist who has to incorporate the drug substance into a formulation together with appropriate excipients. The second very important customer is the plant chemist who has to run the chemical manufacturing process on large scale and ensure that pharmaceutical production receives satisfactory material over the lifetime of the product. To satisfy both clients, all the following aspects (and potentially some more) have to be taken into consideration when designing an API manufacturing process:

- Drug substance quality with respect to purity, polymorph or solvate, residual solvent, and color;

- API powder properties such as particle morphology, particle size and particle size distribution, formulation properties (e.g. compressibility, tablettability among others), bulk density and flowability;

- Chemical manufacturing aspects like throughput and potential bottlenecks (e.g. filtration characteristics, vessel size), scaleability, applicability of standard equipment, solvent amount and toxicity, costs, safety and process robustness.

In the following Sections we will elaborate on some of these aspects and highlight why they are important, along with some recommendation on how to develop robust API manufacturing processes that meet most of the above requirements, constraints and wishes.

\section{Process Design to Ensure Drug Substance Quality}

\subsection{Purity}

Most API manufacturing processes consist of a series of chemical steps followed by a final crystallization that yields the API crystals. In all previous steps, one expects less than $100 \%$ turnover of the starting materials, and routinely sees the formation of byproducts that usually cannot be separated quantitatively. The closer the chemical step is to the final crystallization, the more closely related the byproducts are to the API structure. The separation of byproducts may therefore be extremely difficult in some cases and other purification steps have to be foreseen prior to the final crystallization in order to eliminate these byproducts. Although preparative chromatography or counter current extraction are often used as complementary tools, crystallization has frequently proven to be an extremely powerful tool to separate most unwanted byproducts, and compared to the other techniques mentioned above, it is usually much cheaper.

Given the case that the byproducts are soluble in the mother liquor (which has to be checked) purity depends very much on the crystal growth rate. If the incorporation of the drug substance molecules into the crystal lattice is slow and controlled, byproducts can be prevented from being built into the lattice. However if there is substantial driving force for incorporation, e.g. due to extremely high supersaturation in precipitation processes, then there is a high likelihood that byproducts are also integrated into the crystal lattice, either as molecules or even inside entrapped mother liquor.

Another source of byproduct in the API is mother liquor in the filter cake that is not properly washed out. It is important to distinguish this case from the others in order to find the right development strategy, e.g. by crystallizing coarser material that exhibits less residual solvent in the filter cake and is better accessible to the washing liquor. To find out whether the byproduct is distributed evenly within the crystal or whether it is incorporated or attached only during certain phases of the crystallization process, washing trials can be performed: Significant amounts of substance are washed off stepwise until only a small amount of API remains on the filter. After each wash an HPLC sample is taken and analyzed. This procedure gives insight into potential mechanisms for incorporation and may serve as a guideline to potential process improvements. Due to their similarity to the API, byproducts often have the possibility to act as additives, i.e. they can attach to certain crystal faces and thus lower their growth rate and thereby modify crystal shape [1].

\subsection{Polymorphism and Solvate Formation}

Organic molecules often have the potential to crystallize in various crystalline forms which are called polymorphs if no solvents are included, and solvates if solvents are included in the crystal lattice. For two polymorphs two cases can be distinguished regarding stability: Monotropy if one polymorph is the stable, i.e. least soluble, for all temperatures and enantiotropy if there is a temperature at which the stability ranking changes. In this case - below the so-called transition point - one form is stable and above this temperature the other form is the stable one. Polymorphism for a solvated form is also possible.

Clearly it is extremely important to find out whether different forms exist and if so, which relationship they have regarding stability or if any solvents are involved [2][3]. There are several reasons why these forms have to be carefully characterized:

i) Each form usually has different release characteristics due to different solubility;

ii) A robust crystallization process is only possible if the thermodynamic circumstances are well known and can be taken into consideration (e.g. the transition point in the case of enantiotropy);

iii) Finding as many forms as possible allows the chemist to select the best one (i.e. usually the most stable form under processing and storage conditions) and to obtain patent protection for all known forms.

In order to find multiple crystalline forms intense polymorph screenings are performed usually by varying solvents and process conditions, e.g. temperatures, cooling rates, etc. The role of solvents for 'true' polymorphs is only of kinetic nature - the thermodynamic stability is not influenced by the solvent [4]. However different solvents can interact with certain crystal faces (see Fig. 2 in Section 4.1.) and in this way hinder growth of these faces and thus have the potential to trigger certain polymorphs by suppressing others. Because byproducts can hinder the occurrence of new forms, these screenings should be performed with the purest material available and one should not hesitate to invest into extensive purification before starting such investigations [5-7]. In general solvates are not recommended because their thermodynamic stability is usually a function of temperature and solvent composition which often makes it difficult to produce the correct form and keep it during storage and formulation.

\subsection{Residual Solvents}

There are two main sources for potential issues regarding solvents:

i) Entrapment of mother liquor in the crystal due to extremely fast crystal growth;

ii) Incorporation into the crystal lattice in the case of solvate formation.

The first case can be avoided by taking appropriate design measures in the crystallization procedure. The second case, however, is much more difficult because the solvate incorporation is part of the process and generally registered.

Usually solvates (besides hydrates) cannot be accepted as API for toxicology reasons. Residual solvents are classified according to $\mathrm{ICH}$ guideline Q3C. For toxic solvents very tight limits in the ppm-range are specified and these have to be met in API manufacturing. Therefore the solvent must be removed by drying.

If the transport of solvent molecules within the crystal is hindered, e.g. due to missing channels along which diffusion can take place, drying can be a very timeconsuming process, and easily become the bottleneck of the API manufacturing proce- 
dure. In these cases, reducing particle size may help to reduce drying time, but also extremely low vacuum or good heat transport (e.g. in paddle dryers) may help.

A first good impression about drying kinetics can be obtained by using microbalances that monitor the loss of weight at a certain temperature and pressure. It is rather obvious that this potential problem should be addressed early when choosing the solvent. Ideally one uses non-toxic solvents and avoids solvent mixtures. It is also important to notice that a desolvated solvate may partly loose its stability if the solvent plays a role in the formation of the crystal lattice. In some cases the elimination of the solvent even leads to a breakdown of the crystal lattice, i.e. to amorphization.

\section{Process Design for Optimal API Powder Properties}

\subsection{Particle Morphology}

Morphology is an important factor for the powder behavior in any handling process. Large cubes such as formed by sodium chloride have a very high packing density and show good flow properties. Elongated and fine acicular crystals on the other hand do not pack well and exhibit very poor flow properties. There are thermodynamic and kinetic reasons why a crystal has a preferred growth direction and it is important to understand the underlying principle in order to find a way to modify shape if necessary. An example is shown in Fig. 2 where a crystal structure is depicted that presents the most polar groups at the 110-face.

In Figs. 3-5 the effect of different solvent mixtures on the crystal morphology of this drug substance is shown: the more polar the solvent mixture, the more interaction and thus inhibition of the 110 face is taking place and the shorter the crystals become. In less polar solvent mixtures more elongated crystals are formed.

Using solvents as additives is an elegant way to manipulate crystal shape, but one can also use real additives, which is however not as common in pharmaceutical crystallization because the addition of any product has to be declared and explained to the health authorities.

\subsection{Particle Size and Particle Size Distribution}

Mean particle size and particle size distribution is certainly the most crucial powder property because it has a strong effect on flowability, miscibility, tablettability or

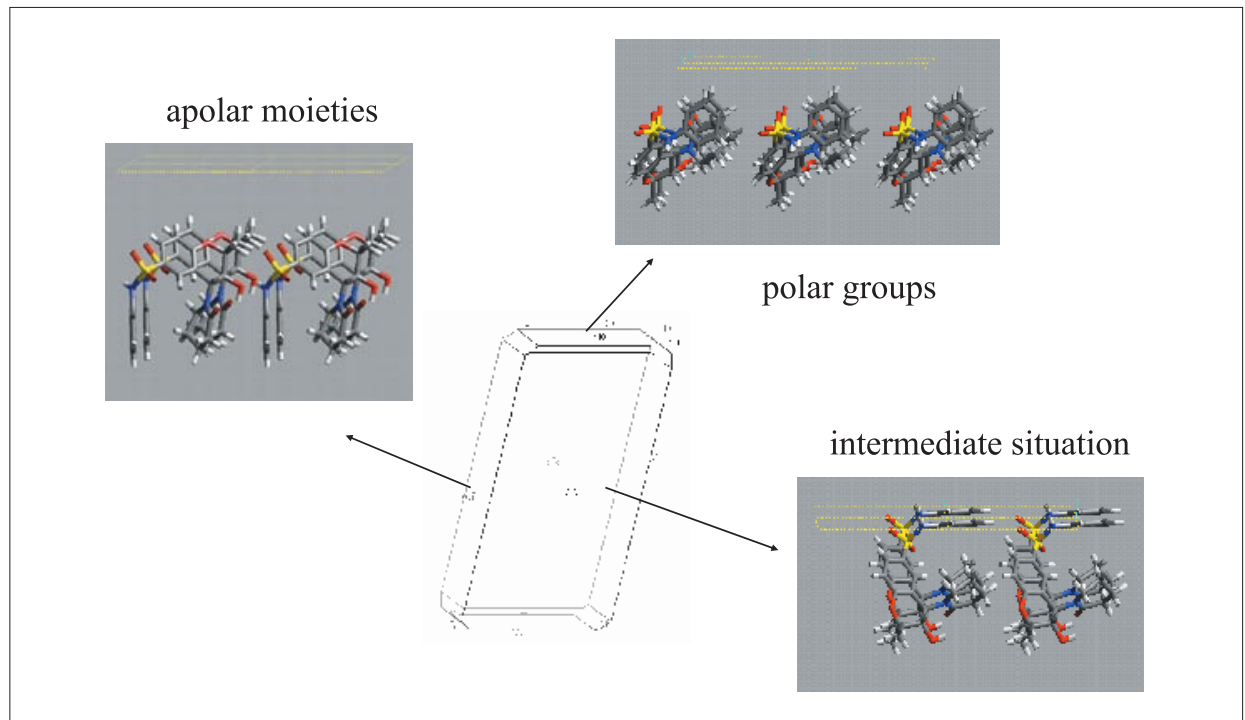

Fig. 2. Crystal faces with different polarity

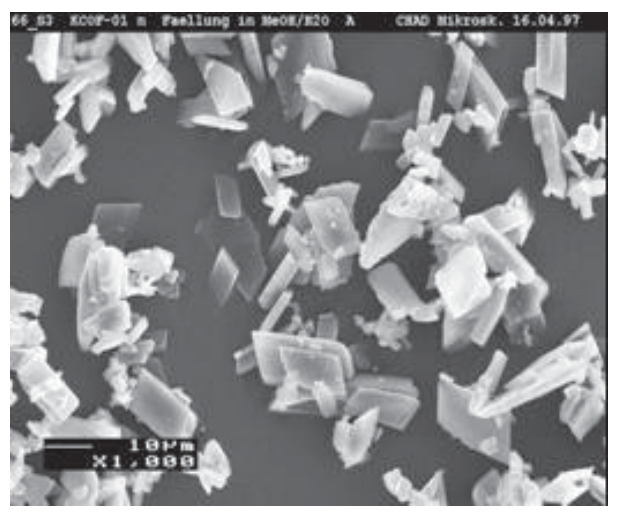

Fig. 3. Crystals obtained from methanol/water

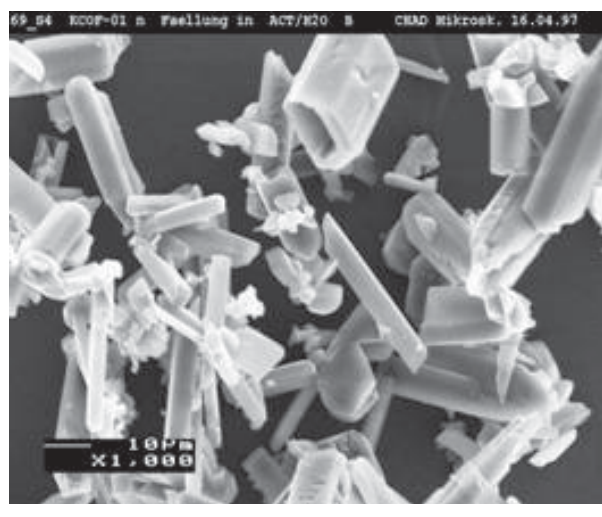

Fig. 4. Crystals obtained from acetone/water processability in general. A broad particle size distribution is usually less desirable because the fines might cause problems due to dust formation or inhibit free flow, whereas too coarse particles might lead to content uniformity problems in tablets or capsules or entail segregation during mixing. Therefore in most cases the target is a more or less monomodal narrow particle size distribution (PSD).

The question often comes down to which PSD best fits the needs of the customer and that need may not be met by a crystallization method. If the drug is used for inhalation, particles have to be around $1-5 \mu \mathrm{m}$ which can hardly be reached directly by any crystallization or precipitation process, thereby necessitating air-jet-milling to reduce the size to the desired range. In this case the size obtained in the crystallization process is not so important - milling will provide the right size distribution. If well-flowing material is required (e.g. for encapsulation) particles have to be large enough to flow well and to allow reproducible dosage into all capsules at high filling speed. Too coarse material however will lead to content uniformity problems depending on the dose range or on the number of crystals present in each capsule.

Let us raise the question how a narrow particle size distribution can actually be obtained. The answer is clear in most cases of application: the primary formation of crystals has to be controlled efficiently and secondary nucleation has to be avoided. Seeding at low supersaturation is a very promising, uncomplicated and efficient method to start the process. Much more difficult to control and therefore not recommended is primary nucleation induced by high supersaturation, e.g. triggered by fast cooling (cold walls) or by antisolvent addition.

Secondary nucleation events are very frequently observed and may severely alter the desired PSD initially foreseen. It occurs when particles collide with other particles, the stirrer blade or the wall at moderate to high supersaturation. This effect can be kept under control if the stirrer speed is chosen appropriately, such as to keep particles sus-

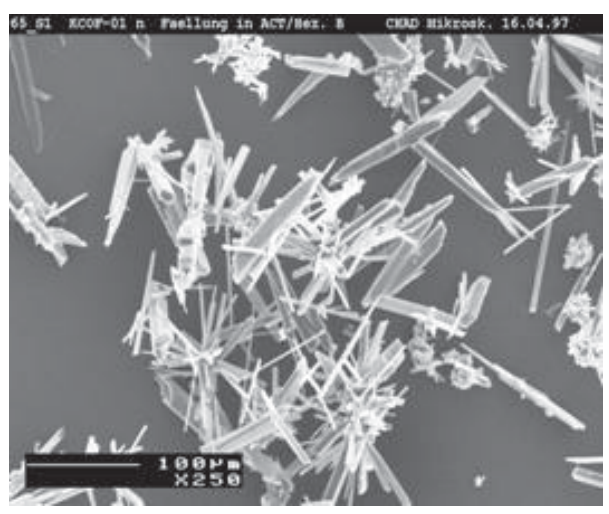

Fig. 5. Crystals obtained from acetone/hexane 
pended, and supersaturation is at a value as low as possible.

\subsection{The Impact of Powder Proper- ties on Drug Product Formulation}

The behavior of API crystals in the formulation can be derived from various intrinsic properties like PSD, shape, surface roughness, elasticity and can strongly depend on external effects like moisture, electrostatic charging, mode of stress, etc. For example flow is a result of the interaction of mass forces $v s$. attractive forces and geometric effects such as particle shape and the design of a hopper or funnel.

Mass forces are given by the size and weight of the particles, and because mass decreases by a power of three with particle size, it is clear that the driving force for flow becomes very poor for small particles. Attractive forces are more difficult to define: Van der Waals forces are always present - they depend very much on the number and area of interparticular or particle-wall contacts, but also on intrinsic material properties like the Hamaker constant or Young's modulus. Plastic deformation can lead to larger contact areas, and hence stronger adhesion forces. Other forces may also be present such as electrostatic charging (e.g. due to triboelectric effects) or liquid bridges due to high moisture or solvents being present. Regarding geometric effects the shape and surface roughness of the drug substance particles may play a role but also the design of the equipment used, which however usually cannot be modified.

Thus to summarize, whenever problems occur in the field of powder processing, it is important to understand the physical reason in order to find the most appropriate solution. In pharmaceutical formulations, a lot can be done by using the right excipients to cover poor drug substance behavior; however, also in this case one should seek a thorough understanding of the underlying physical chemistry in order to avoid surprises when the basic conditions change.

\section{Chemical Manufacturing Aspects}

\subsection{Scale-up}

Scaling API crystallization processes to manufacturing scale is a very demanding task. Basically it is never possible to achieve exactly the same conditions on large scale as on small scale [8][9]. It is well known that the ratio of surface area to volume changes dramatically upon scaleup, and for example high cooling rates that could easily be applied on lab-scale are no longer feasible on production scale. If these were enforced, it would possibly entail in extremely low jacket temperatures and cold reactor walls, and thus may act as a potential source for nucleation or encrustation.
The same applies to most other important parameters in the crystallizer, such as energy input per unit volume, tip speed and recirculation characteristics - one has to decide which parameter is the one to focus on for a given problem - most of the others will automatically be defined consequentially. In the case of large, heavy crystals, the energy input per unit volume may be the appropriate scale-up criterion. However, if the crystals are acicular or very brittle, higher tip speed on production scale may lead to attrition problems as shown in the example of Fig. 6.

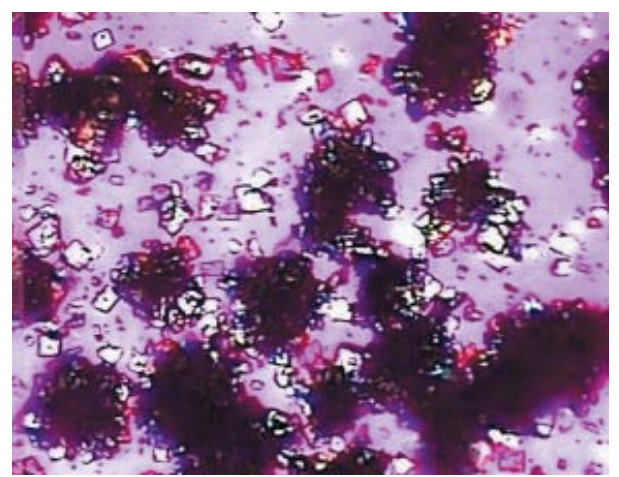

Fig. 6. Abrasion upon scale-up

In this case a slight reduction of the stirrer speed was enough to avoid abrasion, suspension then became borderline - slight demixing on the surface was already observed with these $250 \mu \mathrm{m}$ crystals. If abrasion has to be avoided, one option is to change the stirrer type, e.g. from impeller to pitchedblade, in order to provide sufficient axial entrainment of the particles at low stirrer speed. In extreme cases, if particles up to 1 $\mathrm{mm}$ or above are targeted, air jet crystallizers may be the only option left [10]. Generally, it can be recommended to use lab equipment with geometric similarity to the foreseen plant equipment. This means that the same stirrer type should be used and the same ratio of height to width of the reactor, and of reactor diameter to stirrer diameter has to be chosen. Also baffles and probes inside the reactor should mimic large scale reality.

If mixing is a potential problem, e.g. in precipitation processes, things become even more complicated and one has to assess whether the induction time for nucleation is smaller than the mixing time or vice versa. With this knowledge a suitable up criterion must be defined [11][12].

\subsection{Throughput and Potential \\ Bottlenecks}

Soon after the successful launch of an API manufacturing process, activities are typically initiated to further improve throughput and 'to debottleneck' the limiting steps, if appropriate. As for crystallization a limitation is imposed by the reactor volume, but shorter cycle times may be at- tainable by cooling faster or by shortening temperature holding times. As described above this may lead to severe operational problems if the process is already run at its limits, e.g. when the risk of encrustation due to lower wall temperatures can no longer be excluded. Also secondary nucleation rate will increase if the cooling rate and thus supersaturation is raised, and this will also have an impact on the final PSD. Filtration can be the source of very critical bottlenecks that can sometimes be very hard to overcome. Therefore it is extremely important to assess the role of these processes for the throughput of the whole plant early on. The same is valid for drying. If small portions are dried in tray dryers at very low vacuum in the lab, drying may easily be completed over night. Things may however be drastically different on production scale if $e . g$. a ton of wet material is charged into a large paddle dryer. The required heat of evaporation has to be transferred into the cake and potential abrasion may comminute the particles, especially at early stages when the wet cake does not flow readily and may need extremely high torque on the paddles. Free solvent on the particle surface is usually not the problem in industrial drying process. Small amounts of toxic residual solvents can be extremely difficult to remove to reach the specified solvent content especially because vacuum usually is not as good in the plant as in the lab.

\subsection{Can Standard Equipment be Used or are Special Installations Required?}

Unlike in bulk chemistry, in pharmaceutical production multi-purpose plants are very common with the consequence that the process must be flexible and robust enough to be run in all potential variants available. Therefore it is necessary that all critical steps of the API manufacturing are identified and the potential impact of the equipment used is understood. If, for example, large, sandy crystals have to be crystallized, an anchor stirrer probably would be the wrong choice because it does not provide sufficient axial flow to keep the crystals suspended. Small crystals below $100 \mu \mathrm{m}$ are typically insensitive towards the stirrer used - they do not settle too fast and collisions with the stirrer are rare because they tend to follow the liquid stream lines. Things are different if nucleation is strongly dependant on mixing - in this case special attention has to be paid to the equipment and process parameters used. The same basic knowledge is necessary to assess potential problems in filtration and drying. As described above these two unit operations have a high potential for creating production issues if they are not well investigated and understood. In every case it is worth while contacting 
the plant chemist early enough to see what equipment he intends to use and to consider potential equipment-related limitations already during the design phase of the API manufacturing process.

\subsection{Production Costs}

If the API is to become a large volume product, one needs to consider the potential recovery of the solvents used. The same is valid for chromatographic separation or liquid-liquid extraction where large amounts of solvents are involved. In these cases it is desirable to develop potential scenarios for the workup of solvents or solvent mixtures early on in process development. This point should already be considered during solvent selection. In some cases it may also be worth thinking about API recovery from mother liquors. Even if the recovered material has to undergo additional purification, e.g. by a dedicated crystallization process, it is usually much cheaper than material from the original synthesis.

\subsection{Process Robustness}

Contemplating that API powder properties have a tremendous impact on most drug product manufacturing processes, it is obvious that the API manufacturer has to deliver a consistent particle quality to the formulator. This means that any process must be robust and always yield the desired drug substance quality. As described above, the crystallization process can be very well controlled, if it is designed in such a way that nucleation and crystal growth are actively controlled by the operator. The fewer variations that are possible, the less potential problems can occur.

In retrospect seeding has proven to be the most suitable tool to determine the start of API crystallization processes - it helps to control particle size, polymorphism and solvate formation, purity, downstream processability as well as to a certain extent, shape and yield. Processes with particle formation by spontaneous nucleation are typically far more difficult to control, and their scale-up from lab to pilot, and eventually production scale is difficult. Certain impurities may vanish due to a change in the synthesis or due to a change in the supplier of a precursor and suddenly nucleation may occur much earlier, and at lower supersaturation, leading to a coarser product. Alternatively the kinetic hindrance of a more stable polymorph may no longer be valid and it will suddenly appear. Seeding with the desired (then metastable) form and keeping supersaturation at a low level may even allow the reproduction of the metastable form - it at least minimizes the risk that a new form appears.

As described above the process may have to be run in various plants with slight differences in equipment - anchor stirrer instead of impeller, nutsche instead of centrifuge, double-cone dryer instead of paddle dryer - will it still deliver the right API and what can you do to adjust these changes in order to get the same quality as before? These questions can only be answered if the whole production chain from crystallization, filtration, drying and formulation is really well understood and controlled.

\section{Practical Design of the API Crystallization Process}

The previous sections have dealt with the requirements and design specifications for an API manufacturing process. In this section a pragmatic approach will be given on how to design robust API crystallization processes within a relatively short period of time - a constraint that is typical for pharmaceutical development.

\subsection{Solvent Selection}

The knowledge of the drug substance solubility as a function of temperature is the basis for the design of any crystallization process (green line in Fig. 7).

Cooling is a very easy and thus favorable way of generating supersaturation; therefore an ideal solvent should have high API solubility at elevated temperatures to avoid excessive dilution and low solubility at low temperatures, in order to ensure satisfactory yields. It is important to compare the theoretical yield calculated from these data with the actual yield obtained in the crystallization process. Clearly if theoretical yield is markedly higher, equilibrium has not been reached, which may e.g. be due to kinetically hindered crystallization at low temperatures. In this case, slower cooling or the introduction of holding times at certain temperature levels may be considered. Another important factor is the metastable zone width in the solvent. This is the temperature range by which a saturated solution can be subcooled before nucleation occurs (i.e. the area between solubility and supersolubility curve (dashed orange area in Fig. 7). If this zone is extremely small (e.g. only a few Kelvin) it will be difficult to suppress spontaneous nucleation or to control the crystallization process at all.

As mentioned above, solvents can hinder the growth of certain polymorphs, but they can also have an effect on shape if only certain faces are affected. The last aspects for solvent selection to be mentioned here are price, toxicity and the tendency of the drug to form solvates with any of the solvents used. As a general rule, it is not recommended to choose any solvent that forms solvates, because solvates often cause trouble during process development or later on in chemical production.

\subsection{Generation of Supersaturation}

Supersaturation can be generated by cooling, antisolvent addition, and distillation. Cooling is clearly the most uncomplex, efficient and controllable of the three. Undercooling can be avoided by jacket control and slow cooling rates. There is no change in solvent composition and hence no high risk for solvate problems.

If the solubility curve is too flat, one can consider adding antisolvent prior to seeding and cooling but try to avoid adding the antisolvent during the crystallization process for the reasons given below. Antisolvent addition cannot be avoided in the case of precipitations where extremely high nucleation rates are sought for; however, in normal crystallization processes addition of antisolvents should be avoided for the following reasons:

i) The risk of solvate formation and potential complexity increases with the number of solvents involved;

ii) Crystals will be affected by the changing environment during the crystallization process - growth rates, nucleation

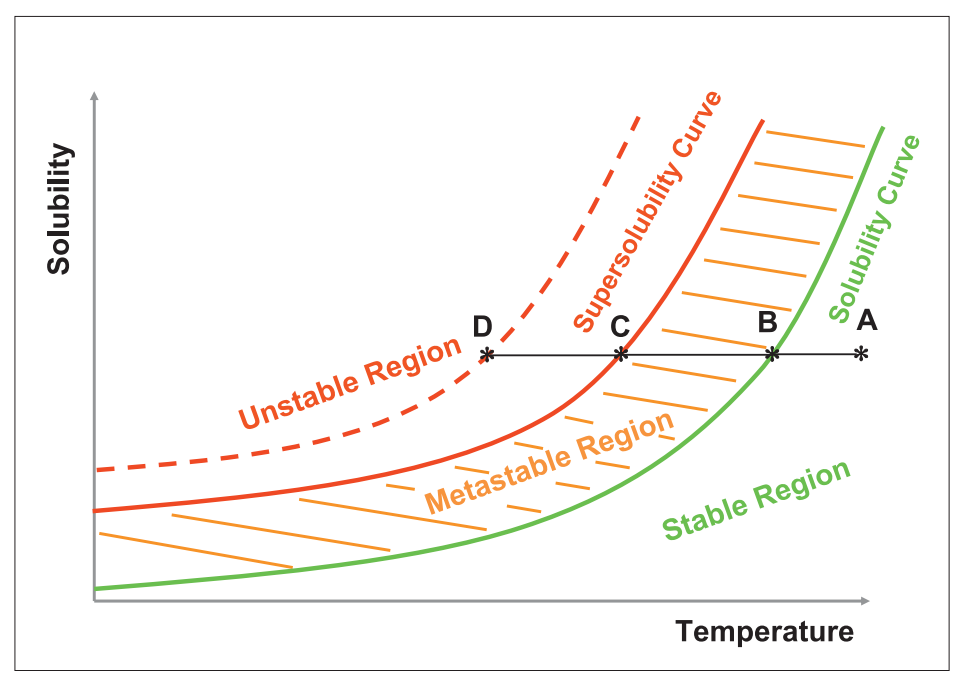

Fig. 7. Solubility curve and supersolubility curve 
mechanisms and even thermodynamic regimes may change, i.e. the stability ranking of solvates may change during antisolvent addition. Due to high supersaturation oiling-out may happen;

iii) Most critical is the addition point of the antisolvent in the reactor - here is the highest local supersaturation and precipitation may happen even if seed crystals are present. If so, often the kinetically favored form will appear, not the thermodynamically stable one. Due to the high crystal growth rate impurities are more likely to be incorporated.

All these potential issues make the scaleup of anti-solvent addition processes very demanding task.

Distillation is even more challenging and therefore not recommended at all - in the case of solvent mixtures a wide range of solvent compositions will be run through with enormous potential for solvate surprises. Furthermore due to the decreasing liquid

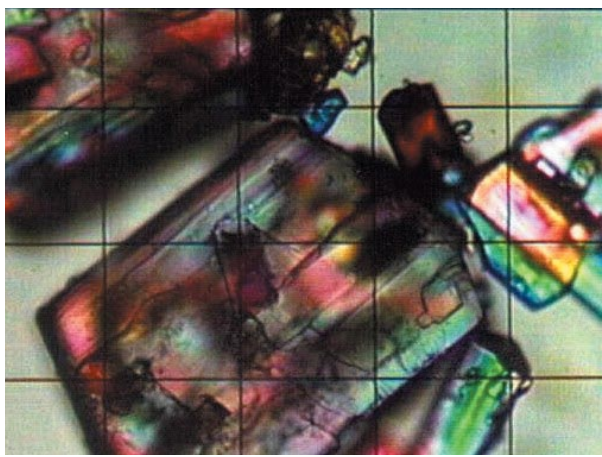

Fig. 8. Spontaneous nucleation

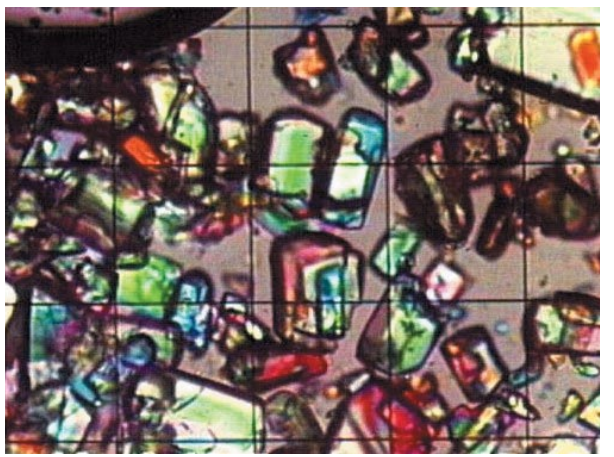

Fig. 9. Seeded with $0.1 \%$ seeds

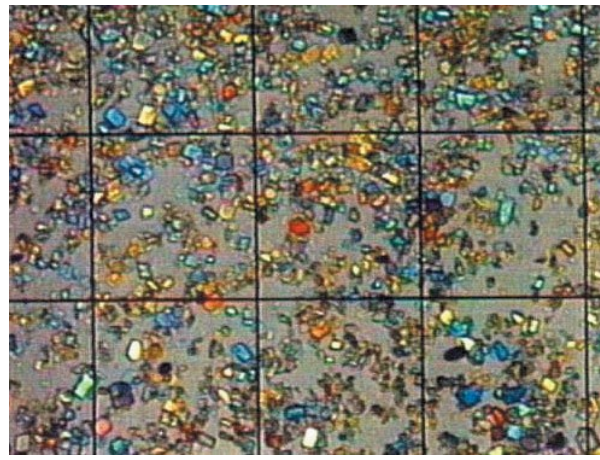

Fig. 10 Seeded with $1 \%$ seeds. Grid dimension in all figures: $100 \mu \mathrm{m}$ level in the reactor crusts may form on the walls that can fall into the crystallizer and inoculate with unwanted forms.

\subsection{Inoculation/Seeding}

Seeding is by far the most recommendable tool to start crystallization [13]. If it is performed at low supersaturation, uncontrolled spontaneous nucleation events can be suppressed. A given number and size of seeds determine the final particle size. To estimate the mass of seeds $M$ needed to obtain a certain particle size $\mathrm{L}$ the following formula can be used [14]:

$$
\mathrm{M}_{\text {seed }}=\mathrm{M}_{\text {final }} \times\left(\mathrm{L}_{\text {seed }} / \mathrm{L}_{\text {final }}\right)^{3}
$$

Figs 8-10 show an example where particle size has been manipulated just by adding different amounts of micronized seeds (mean size around $3 \mu \mathrm{m}$ ) with the intention to produce finer crystals with better drying behavior.

The possibility to adjust particle size is not the sole advantage of seeding. It also enables the initial crystal growth rate to be kept under control if it is performed at low supersaturation and thus it helps to achieve high purity. Furthermore seeding with the desired polymorph or solvate can help to harvest the correct form reproducibly and to suppress the formation of amorphous material which is usually undesired for stability and manufacturing reasons [15][16]. Attention must be paid when seeding with desolvated solvates is tackled, i.e. in cases where the drug substance crystallizes as a solvate which is then desolvated upon drying. The dry API may not be effective as seed material and may have to be reconstituted in the solvent to its solvated form prior to seeding.

The biggest advantage of seeding is the process robustness obtained with this method. The process has hardly any chance to become uncontrolled if seeding is well done. Deviations may arise from the use of too few seeds. Seeding a $500 \mathrm{~kg}$ batch with $50-100 \mathrm{~g}$ of seeds should be referred to as 'homeopathic' seeding, literally. If the surface provided by the seeds is not large enough to consume the provided supersaturation over time, then supersaturation is further built up, and additional spontaneous nucleation may be observed although seeds are present. Furthermore, it is clear that, even if crystallization is fast enough to cope with this amount of material, the purity obtained will probably be suboptimal due to an extremely high crystal growth rate. As a rule of thumb around $0.1-1 \%$ of seeds are recommended. Another source of potential problems can be the addition of dry seed material directly into the crystallizer containing the supersaturated solution. Especially if the material has been stored over months or years it may have solidified and may not be distributed evenly in the reactor. This decreases the effective seed surface provided for the crystallization and thus adversely affects the final product PSD. The result can be similar as in the case of homeopathic seeding - at least it will scatter more than necessary. The best practice is to suspend the crystals in the solvent using high-shear equipment to ensure sufficient dispersion and then add this dispersion to the crystallizer. More specific information may be found in the overview article by Heffels and Kind [14].

\subsection{Further Processing}

After seeding, it is worthwhile to wait for some time in order to allow the seeds to grow. This will usually take $30 \mathrm{~min}$ to $1 \mathrm{~h}$ until equilibrium is more or less reached, and then the suspension can be cooled down further. If cooling is initiated immediately after seeding, there is a danger that the small surface area provided for desupersaturation by the seeds may not be sufficient, to take up this additional amount of material thus favoring spontaneous nucleation. This is also the reason why cooling in general should not be performed too fast; $0.1-0.2$ $\mathrm{K} / \mathrm{min}$ is typically a reasonable range. Note that also secondary nucleation is depending on the supersaturation and may be dramatically increased if cooling is too fast. During the design of a crystallization process one should test higher and lower cooling rates in order to find out where the limitations for the particular drug substance are. Probably the best cooling strategy is to cool slowly after seeding when the seed surface is still small and the solubility curve is steep (see Fig. 7). The further crystallization advances the faster cooling can be. The resulting curve is similar to a negative parabola, but it can be approximated by two or three linear ramps (dashed green line in Fig. 11). In this case supersaturation can nearly be kept

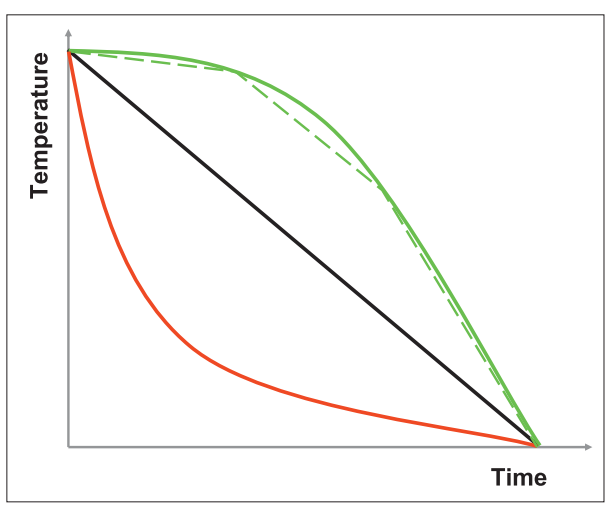

Fig. 11. Variants for cool down after seeding: red line - natural cooling, black line - linear cooling, green line - controlled parabolic cooling and green dashed line - approximated parabolic cooling 
constant during the process which ensures optimal crystal quality [17].

In no case should natural cooling be applied. As mentioned above, in some cases it can be beneficial to hold the temperature for a while or decrease the cooling rate at lower temperatures in order to reach a real equilibrium and not get trapped in the ' $\mathrm{ki}$ netic trap', i.e. when crystallization kinetics at low temperatures (around and below $0{ }^{\circ} \mathrm{C}$ ) is sometimes so slow that even long holding times are not enough to reach equilibrium concentration, and thus optimal yield. Generally speaking the stirrer speed should be kept as slow as possible to avoid secondary nucleation.

\subsection{Filtration}

It is important to check at an early stage whether filtration is a potential problem for production. Filtration times on the large scale can be calculated from small-scale experiments even if usually not all aspects can be taken into consideration (e.g. compressibility on a centrifuge if only standard nutsche experiments are available). As a rule of thumb, cake formation time scales quadratically with cake height -i.e. if a 1 $\mathrm{cm}$ cake height takes $1 \mathrm{~min}$ to form in the lab, $20 \mathrm{~cm}$ on a large-scale filter will need $400 \mathrm{~min}$ or nearly $7 \mathrm{~h}$. Washing time scales linearly with cake height, i.e. if the $1 \mathrm{~cm}$ lab-cake needs $5 \mathrm{~min}$ for the wash, it will be $100 \mathrm{~min}$ in the plant, and so on. In this example filtration will take more than $8 \mathrm{~h}$ which still may be o.k. if the whole batch can be filtered in one portion. If however six centrifuge portions are foreseen, the total filtration time sums up to $50 \mathrm{~h}$ which is usually not acceptable, at least for largevolume products. Problems may multiply in the case of compressible filter cakes.

\subsection{Drying}

As mentioned above it is important to understand if drying is a potential issue or can become the bottleneck of the API man- ufacturing process. In case of solvates it is necessary to assess how easily the solvent can be removed from the crystal lattice, and whether there is a stable parameter plateau where the desired solvate (hydrate) can be obtained. Thermogravimetry is a good tool for these investigations, especially if it can be performed under vacuum (e.g. the Rubotherm Magnetic Suspense Balance). Only pressure values that are realistic for large-scale production should be applied (i.e. not below 20 mbar) in order to be on the safe side for production-scale operation. Crystal structure analysis can help to understand how the solvent molecules are entrapped in the lattice and whether there are open channels for release by diffusion. Potential drying problems can be addressed, e.g. by using special equipment like agitated driers, powerful roots blowers, special process conditions (preheating before pulling vacuum) etc. If agitated driers are foreseen, it should be investigated whether attrition has an effect on the drug substance properties as in the example shown in Fig. 12.

\section{Summary}

Designing a manufacturing process for an API is a challenging task. The physical parameters required for the drug manufacture have to be defined and translated into a crystallization and work-up process. There are many potential hurdles that have to be overcome - first of all polymorphism and solvate formation, but also uncontrolled nucleation and the incorporation of byproducts. It is important to generate a good understanding of a particular drug substance, its behavior in the whole process chain, its 'weaknesses' and 'strengths'. Only if all relevant aspects are taken into consideration one can design a good manufacturing process and be sure that it is really scaleable and robust. Unfortunately only very little of the experience gained can be trans-

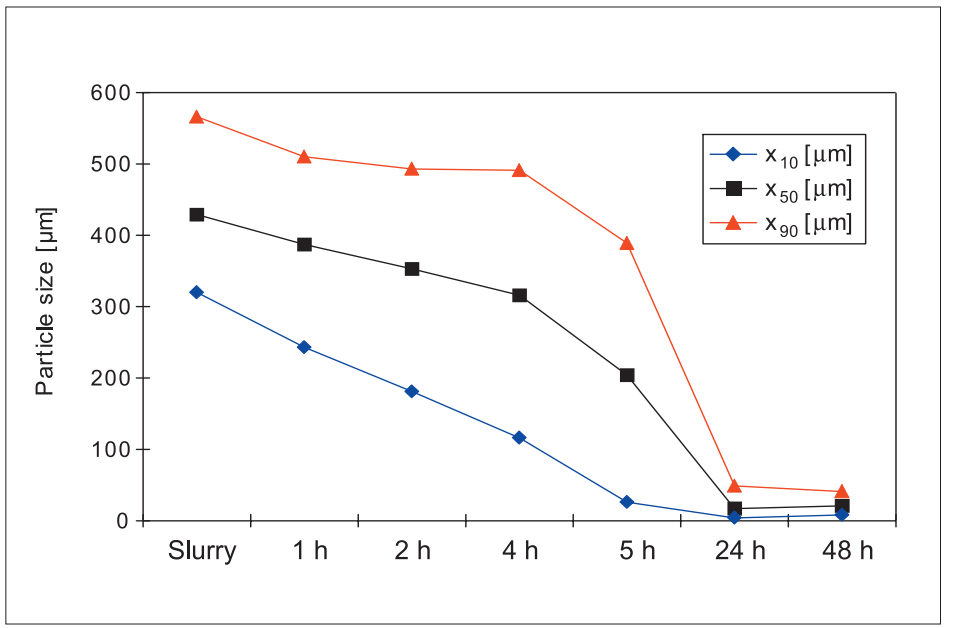

ferred to other APIs - even minute changes in the molecule can dramatically change the crystal lattice and all kinetic and thermodynamic characteristics. This variability makes the crystallization of APIs an everlasting challenge, however if the described principles are applied, the risk for failure can be minimized, albeit unfortunately never excluded.

Received: May 23, 2006

[1] J. Nyvlt, J. Ulrich, 'Admixtures in Crystallization', $\mathrm{VCH}, 1995$, Weinheim.

[2] T.L. Threlfall, Analyst 1995, 120, 24352460.

[3] A. Burger, R. Ramberger, Mikrochimica Acta 1979, 2, 259-271.

[4] S. Khoshkhoo, J. Anwar, J. Phys.D: Appl. Phys. 1993, 26, B90-B93.

[5] FDC Quality Control Records 1996, 30, 1.

[6] W.C. McCrone, in 'Physics and Chemistry of the organic solid state', Ed. D. Fox, M.M. Labes, A. Weissberger, Interscience, 1965, New York and London, 726-767.

[7] J.D. Dunitz, J. Bernstein, J. Acc. Chem. Res. 1995, 28, 193-200.

[8] J.Y. Oldshue, in 'Handbook of Industrial Crystallization', Ed. A.S. Myerson, Butterworth-Heinemann, 1993, p.167.

[9] W.J. Genck, Chem. Eng. Prog. 2003, 99, 36-44.

[10] B. Braun, H. Grön, J. Tschernjaew, Crystal Growth and Design 2004, 4, 915-920.

[11] O. Söhnel, J. Garside, 'Precipitation, Basic Principles and Industrial Applications', Butterworth-Heinemann, 1992, Oxford.

[12] Literature on the fundamentals of precipitation processes is abundant, $c f$. e.g. the textbook of O. Söhnel and J. Garside.

[13] W.P. Moore, Chem. Eng. Prog. 1994, 90, 73-79.

[14] S.K. Heffels, M. Kind, Proceed. 14th Intern. Symposium on Industrial Crystallization in Cambridge 1999, 14, 2234-2246.

[15] For more details on amorphous formation and significance, $c f$. the work of Hancock and Zografi.

[16] B.C. Hancock, G. Zografi, Pharm. Sci. 1997, $86,8$.

[17] J. Nyvlt, J.W. Mullin, Chem. Eng. Sci. 1971, 26, 369-377.

Fig. 12. Effect of attrition on particle size during drying in a paddle dryer 